\title{
Sex effects on short-term complications after hip fracture: a prospective cohort study
}

This article was published in the following Dove Press journal:

Clinical Interventions in Aging

5 August 2015

Number of times this article has been viewed

\author{
Wilhelmina Ekström ${ }^{1}$ \\ Bodil Samuelsson ${ }^{2}$ \\ Sari Ponzer ${ }^{3}$ \\ Tommy Cederholm ${ }^{4,5}$ \\ Karl-Göran Thorngren ${ }^{6}$ \\ Margareta Hedström ${ }^{7}$ \\ 'Department of Molecular Medicine \\ and Surgery, Section of Orthopaedics \\ and Sports Medicine, Karolinska \\ Institutet, Karolinska University \\ Hospital, Solna, ${ }^{2}$ Department of \\ Clinical Sciences, Karolinska Institutet, \\ Danderyd Hospital, ${ }^{3}$ Department \\ of Clinical Science and Education, \\ Section of Orthopaedics, Karolinska \\ Institutet, Södersjukhuset, Stockholm, \\ ${ }^{4}$ Department of Public Health and \\ Caring Sciences, ${ }^{5}$ Department of \\ Geriatrics, Uppsala University, \\ Uppsala University Hospital, \\ Uppsala, ${ }^{6}$ Department of Clinical \\ Sciences, Lund, Orthopaedics, Lund \\ University, Lund, 'Department \\ of Orthopaedics, Institute of Clinical \\ Science, Intervention and Technology \\ (CLINTEC), Karolinska University \\ Hospital, Huddinge, Stockholm, \\ Sweden
}

Objectives: To evaluate potential sex differences and other factors associated with complications within 4 months after a hip fracture.

Methods: A total of 1,915 patients $\geq 65$ years ( 480 men) with hip fracture were consecutively included in a prospective multicenter cohort study. A review of medical records and patient interviews according to a study protocol based on the Standardized Audit of Hip Fractures in Europe (SAHFE, RIKSHÖFT) was performed. Sex differences in comorbidity according to the American Society of Anesthesiologists score and complications 4 months after a hip fracture were registered. Multivariate logistic regression analysis was performed to identify factors related to complications.

Results: Male sex was associated with worse general health according to the American Society of Anesthesiologists classification $(P=0.005)$ and with more comorbidities $(P<0.001)$. Male sex emerged as a risk factor for developing pneumonia $(P<0.001)$, and additionally, $18 \%$ of the men suffered from cardiac complications compared with $13 \%$ of the females $(P=0.018)$. Female sex was predisposed for urinary tract infections, $30 \%$ vs $23 \%$ in males $(P=0.001)$. Mortality was higher in the male vs female group, both within 30 days ( $15 \%$ vs $10 \%, P=0.001)$ and at 4 months $(24 \%$ vs $14 \%, P=0.001)$. Conditions associated with pneumonia were male sex, pulmonary disease, and cognitive impairment. Cardiac complications were associated with delayed surgery and cardiovascular and pulmonary disease.

Conclusion: Before surgery, men with hip fracture already have a poorer health status and higher comorbidity rate than women, thus resulting in a twofold increased risk of pneumonia. Cognitive dysfunction and pulmonary disease contributed to pneumonia in men. Delayed surgery seems to increase the risk for cardiac complications. It is important to consider the sex perspective early on together with cardiopulmonary comorbidity and cognitive dysfunction to be able to counteract serious complications that may lead to death.

Keywords: hip fracture, male sex, complications, associated factors

\section{Introduction}

Patients with hip fracture are commonly encountered in orthopedic and geriatric wards. ${ }^{1}$ These patients often suffer from several comorbidities ${ }^{2}$ and therefore require considerable nursing and medical care, in addition to the specific care needed for the hip fracture. Presence of comorbidities increases the risk of complications and frequently causes increased suffering with prolonged hospital stay ${ }^{3-5}$ and increased mortality rate. ${ }^{6}$ Few studies have been conducted with large samples to examine sex differences with focus on the effects of specific risk factors for short-term serious complications. It has been found that men more often suffer comorbidities at the time of hip fracture, that they are twice as likely to die after a hip fracture compared to women, and that the cause of death is dominated by infectious diseases such as pneumonia, influenza, and septicemia. ${ }^{7}$ Another study has confirmed that men are more likely to die from
Correspondence: Wilhelmina Ekström Department of Molecular Medicine and Surgery, Section of Orthopaedics and Sports Medicine, Karolinska Institutet, Karolinska University Hospital, Solna, I7I76 Stockholm, Sweden

Tel +46 851775759

Email wilhelmina.ekstrom@karolinska.se 
respiratory disease, malignant neoplasm, and circulatory disease compared to women. ${ }^{8}$ However, Endo et $\mathrm{al}^{9}$ found that there was no difference across sex when considering the number of comorbidities prior to surgery, but men had a worse physical status when assessed according to the American Society of Anaesthesiologists (ASA) score. In the same study, men had a higher proportion of one or more postoperative complications, with particular focus on pneumonia, arrhythmia, delirium, and pulmonary embolism. ${ }^{9}$

In 2009, we published a study analyzing sex differences in patients with a hip fracture and found that cognitive function in men was strongly associated with a higher risk of loss of walking ability and mortality. ${ }^{10}$ In another report from the same population, we analyzed associated factors for mortality in men and women with a hip fracture and presented a prognostic model including age, sex, ASA, and cognitive function. ${ }^{11}$

Considering the serious complications that could occur after hip fracture surgery, especially in men, it would be of great value to better understand factors related to postoperative complications at admission in order to offer optimal care for patients at high risk.

The aim of the present study was to analyze sex differences and other possible factors associated with short-term complications. In contrast to our previous reports from this cohort, this report focuses on perioperative complications and possible associated factors within 4 months after surgery.

\section{Materials and methods}

All patients admitted with a hip fracture to any of the four university hospitals in Stockholm were consecutively included in a prospective cohort study throughout $2003 .^{10}$ The patients were enrolled for 1 year and then followed for 2 years. Patients with pathologic hip fractures, such as metastatic fractures, malign and benign bone cyst, and fracture due to Paget's disease, and patients younger than 65 years were excluded. The overall aim of the initial study was to describe the population with hip fracture in a geographically defined urban area and to evaluate the effect of cognitive function and sex on functional outcome. ${ }^{10}$ This study aims to further evaluate the sex effect on complications within 4 months after surgery. Patients were treated according to the Swedish National Guidelines. ${ }^{12}$ The guidelines recommend surgery within 24 hours, and encouraged early mobilization, and restricted time for the routine use of perioperative urinary catheter. Data collection was performed by a trained research nurse at each hospital and was then confirmed by the senior orthopedic consultant responsible for the study. At inclusion, the research nurse made a review of the medical records and carried out a structured interview with each patient according to the study protocol based on the national register RIKSHÖFT-standardized audit of hip fractures in Europe (SAHFE). ${ }^{13}$ Data collection was performed at baseline and at 4 months. Proxy respondents were interviewed if the patients were unable to provide reliable answers.

Each patient's physical and mental status on admission to the hospital were regarded as baseline. The index episode was defined as the patient status during the time period from admission to discharge from the hospital. Patient interviews were carried out during the index episode and at follow-up after 4 months.

The patient's current residence status was defined as living in own home or in an institution, which would include nursing homes or other staffed facilities, for example. Activities of daily living (ADL) status was recorded according to the Katz ADL Index. ${ }^{14}$ The Katz ADL index status is based on an evaluation of the functional dependence or independence of patients in bathing, dressing, going to the toilet, transferring, continence, and feeding. ADL index A indicates independence in all six functions, index $\mathrm{B}$ independence in all but one of the six functions. Indexes $\mathrm{C}-\mathrm{G}$ indicate dependence in bathing and at least one more function. Presence or absence of concomitant diseases (comorbidity) was based on the medical history obtained from the patient, a proxy, and the medical records. Comorbidities were classified as cardiovascular disease, stroke, pulmonary disease, renal disease, diabetes mellitus, rheumatoid arthritis, Parkinson disease, and malignancy. The patient's smoking status was recorded as "current smoker" or "nonsmoker". Experienced orthopedic surgeons classified the hip fractures according to the SAHFE protocol. ${ }^{13}$ The attending anesthesiologist assessed the patient's health status using the ASA score. ${ }^{15}$ Cognitive status was assessed during the index period using the Short Portable Mental Status Questionnaire (SPMSQ), which is a 10 -item questionnaire. A range of 0-2 correct answers indicates severe cognitive dysfunction, 3-5 moderate dysfunction, 6-7 mild dysfunction, and 8-10 lucid. ${ }^{16}$ For the purpose of this study, we dichotomized the answers into two categories: cognitive dysfunction (ie, 0-7 correct answers) or no cognitive dysfunction (8-10 correct answers). In cases where the test was not conducted, patients were classified as having cognitive dysfunction if they had a diagnosis or known history of dementia; otherwise they were recorded as missing. Time to surgery was registered in days from admission to the hospital. 
Pressure ulcers were classified as grades I-IV according to the European Ulcer Advisory Panel (EPUAP 1999). ${ }^{17}$ The following medical and general complications, as defined in the SAHFE protocol, ${ }^{13}$ were used for the purpose of this study: pneumonia, cardiac complications (cardiac failure, arrhythmia, angina), deep venous thrombosis, pulmonary embolism, wound infection (superficial and deep), urinary tract infection (UTI), acute renal failure, gastrointestinal bleeding, myocardial infarction, cerebrovascular lesion, and pressure ulcer.

Complications were identified by collecting information from medical records. Pressure ulcers were recorded on admission, during initial hospital stay, and at the time of discharge. Information on mortality was obtained from each hospital's discharge register or from a proxy.

\section{Statistical methods}

Data were presented as mean $\pm \mathrm{SD}$ (standard deviation) or as indicated. Categorical and nominal data were summarized as percentages (within sex group) and tested for sex differences with the $\chi^{2}$ test. The crude association between baseline variables and the most frequent complications is described and presented as odds ratios (ORs), with $95 \%$ confidence interval (CI), and $P$-values. Associated variables were entered into logistic regression models using the stepwise selection procedure, one model for each selected complication. All variables in the regression models were tested for interactions. All results are presented as ORs with their respective 95\% CIs. All tests were two-sided, with the level of significance set at $P<0.05$. Analyses were performed with IBM SPSS Statistics 19 (IBM Corporation, Armonk, NY, USA).

The study was conducted according to the Helsinki Declaration, and the local ethics committee approved all protocols.

\section{Results}

Of the initial 2,134 patients with a history of hip fracture, 179 patients were younger than 65 years and hence excluded from the study. The study sample consisted of 1,955 elderly patients. Of them, 12 patients died before surgery. Interview data were missing at baseline for an additional 28 patients, leaving just 1,915 patients (1,435 women and 480 men) in the final analysis. Baseline demographics, fracture type, and medical conditions are presented by sex in Table 1 .

\section{Comorbidity - sex differences at baseline} Mean age was higher in the female population, at $84 \pm 6.8$ vs $82 \pm 7.4$ years in men $(P<0.001)$. Male sex was associated with a higher percentage ASA $3-4,69 \%$ vs $61 \%$ in female $(P=0.002)$. Men also had more comorbidities, mean, 1.6 (SD, 1.08) compared to 1.4 (SD, 0.96$)$ in women $(P<0.001)$. Cardiovascular disease was found to be $66 \%$ among men and $67 \%$ among women $(P=0.824)$. Significantly more men had a history of stroke ( $22 \%$ men vs $14 \%$ women, $P<0.001)$, pulmonary disease ( $20 \%$ men vs $15 \%$ women, $P=0.002)$, Parkinson disease ( $5 \%$ men vs $3 \%$ women, $P=0.017$ ), renal disease ( $6 \%$ men vs $4 \%$ women, $P=0.038$ ), and malignancy ( $21 \%$ men vs $14 \%$ women, $P<0.001$ ). In the male group $14 \%$ were suffering from diabetes mellitus vs $11 \%$ in the female group ( $P=0.097)$. Rheumatoid arthritis was found to be $5 \%$ among men and 7\% among women $(P=0.169)$. Fifty-nine percent of males and $58 \%$ of females had a cognitive dysfunction, according to the SPMSQ ( $P=0.594)$ (Table 1).

\section{Complications - sex differences}

The overall complication rate was $59 \%$ in the male group vs $56 \%$ in the female group ( $P=0.119)$. About $14 \%$ of male patients suffered from pneumonia compared to $6 \%$ women $(P<0.001)$, and $18 \%$ of the men had a cardiac complication compared to $13 \%$ of the women $(P=0.018)$. Female sex predisposed to UTI, $30 \%$ vs $23 \%$ in males $(P=0.001)$. Mortality was higher in the male group when compared with women, both within 30 days ( $15 \%$ vs $10 \%$, respectively, $P=0.001)$ and at 4 month follow-up ( $24 \%$ vs $14 \%$, respectively, $P=0.001$ ) (Table 2).

\section{Associations between baseline variables and complications}

Sex, age, ASA, time-to-surgery, cardiovascular or pulmonary disease, and cognitive function emerged as significantly associated with complications. In the crude analysis, cognitive dysfunction had a negative association with pressure ulcer, cardiac complication, and pneumonia (Table 3). A history of stroke did not correlate with any of the complications. A similar lack of correlation was found for a history of diabetes mellitus.

Patients with pneumonia had 2.6 times higher odds (OR: 2.6, 95\% CI: 1.7-3.9, $P<0.001)$ and patients with cardiac complications 3.9 higher odds (OR: 3.9, 95\% CI: 2.9-5.4, $P<0.001)$ of increased 30-day mortality. At 4 months, the mortality was still 2.2 times more likely in patients with pneumonia (OR: $2.2,95 \% \mathrm{CI}: 1.5-3.2, P<0.001$ ) and 2.5 times more likely in patients with cardiac complication (OR: 2.5, 95\% CI: 1.8-3.3, $P<0.001$ ) (Table 3).

The effect of the status at baseline on the four most common complications was analyzed in a stepwise logistic regression 
Table I Baseline demographics in I,915 patients aged $>65$ years with a hip fracture

\begin{tabular}{|c|c|c|c|c|c|}
\hline & \multicolumn{2}{|l|}{ Women } & \multicolumn{2}{|l|}{ Men } & \multirow[t]{2}{*}{$P^{*}$} \\
\hline & $\mathbf{N}$ & $\%$ & $\mathbf{N}$ & $\%$ & \\
\hline Age, mean (SD) & $84(6.8)$ & & $82(7.4)$ & $65-101$ & $<0.001$ \\
\hline \multicolumn{6}{|l|}{ Residence $^{\mathrm{a}}$} \\
\hline Own home & I,077 & 76 & 373 & 80 & 0.153 \\
\hline Institution & 334 & 24 & 96 & 20 & \\
\hline \multicolumn{6}{|l|}{ Activities of daily living ${ }^{b}$} \\
\hline Katz A-B & 986 & 73 & 312 & 70 & 0.138 \\
\hline Katz C-G & 357 & 27 & 135 & 30 & \\
\hline \multicolumn{6}{|l|}{ Type of fracture ${ }^{c}$} \\
\hline Cervical & 668 & 48 & 255 & 53 & 0.004 \\
\hline Pertrochanteric & 655 & 46 & 181 & 38 & \\
\hline Subtrochanteric & 92 & 6 & 44 & 9 & \\
\hline \multicolumn{6}{|l|}{ ASA score ${ }^{d}$} \\
\hline ASA I-2 & 557 & 39 & 147 & 31 & 0.002 \\
\hline ASA 3-4 & 872 & 61 & 328 & 69 & \\
\hline \multicolumn{6}{|l|}{ Number of comorbidities } \\
\hline Mean, (SD), median & I.4 (0.96) & I & $1.6(1.08)$ & 2 & $<0.001$ \\
\hline $0-1$ & 847 & 60 & 230 & 48 & \\
\hline$\geq 2$ & 568 & 40 & 247 & 52 & $<0.001$ \\
\hline \multicolumn{6}{|l|}{ Specific comorbidity } \\
\hline Cardiovascular disease & 951 & 67 & 317 & 66 & 0.824 \\
\hline Stroke & 230 & 14 & 94 & 22 & $<0.001$ \\
\hline Pulmonary disease & 206 & 15 & 97 & 20 & 0.002 \\
\hline Renal disease & 52 & 4 & 28 & 6 & 0.038 \\
\hline Diabetes & 156 & II & 66 & 14 & 0.097 \\
\hline Rheumatoid arthritis & 100 & 7 & 25 & 5 & 0.169 \\
\hline Parkinson's disease & 37 & 3 & 23 & 5 & 0.017 \\
\hline Malignancy & 198 & 14 & 100 & 21 & $<0.001$ \\
\hline Cognitive dysfunction ${ }^{f}$ & 775 & 58 & 262 & 59 & 0.594 \\
\hline \multicolumn{6}{|l|}{ Pressure ulcer at admission } \\
\hline Grade I & 54 & 4 & 14 & 3 & 0.392 \\
\hline Grade II-IV & 52 & 4 & 17 & 4 & 0.944 \\
\hline $\begin{array}{l}\text { Surgery same day } \\
\text { or day after admission }\end{array}$ & 1,092 & 76 & 357 & 74 & 0.446 \\
\hline
\end{tabular}

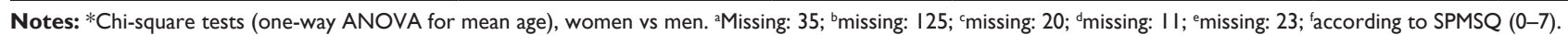

Abbreviations: ASA, American Society of Anesthesiologists; SD, standard deviation; SPMSQ, Short Portable Mental Status Questionnaire; ANOVA, analysis of variance.

analysis (Table 4). Female sex posed a higher risk for UTI, whereas male sex was associated with a higher risk of pneumonia. Cardiac complications did not associate with sex. Cognitive dysfunction, present pulmonary disease, and male sex were the strongest factors related to the development of pneumonia. Surgical delay was associated with cardiac complications and increased incidence of developing pressure ulcer.

\section{Discussion}

In this study, we confirm that before surgery, men with hip fracture already have a poorer health status and higher comorbidity rates than do women, resulting in a twofold increased risk of pneumonia and also an increased risk of cardiac complications.

Cognitive dysfunction increased the risk of any kind of complication and was, together with present pulmonary disease, a dominating factor related to pneumonia in men. Delayed surgery seems to increase the risk for cardiac complications and should be avoided if possible. It is important to consider the sex perspective early on together with cardiopulmonary comorbidity and cognitive dysfunction to be able to counteract serious complications that may lead to death.

The relatively high complication rate compared to other similar studies may be explained by our approach of including all patients admitted for a hip fracture; in other words, that we also included patients with cognitive dysfunction. Cognitive dysfunction is known to be associated with a negative outcome after a hip fracture. The SPMSQ 10-item questionnaire has been reported to provide relevant information about the cognitive status of patients with hip fractures. ${ }^{18}$ We have previously reported the negative impact of severe cognitive dysfunction on mortality. ${ }^{11}$ Patients with mild to 
Table 2 Complications $0-4$ months after a hip fracture in I,915 patients aged $\geq 65$ years

\begin{tabular}{|c|c|c|c|c|c|}
\hline & \multicolumn{2}{|c|}{ Women } & \multicolumn{2}{|c|}{ Men } & \multirow[t]{2}{*}{$P *$} \\
\hline & $\mathbf{N}$ & $\%$ & $\mathbf{N}$ & $\%$ & \\
\hline \multicolumn{6}{|l|}{ Number of complications } \\
\hline None & 613 & 44 & 194 & 42 & 0.119 \\
\hline One & 500 & 35 & 156 & 33 & \\
\hline Two or more & 290 & 21 & 118 & 25 & \\
\hline \multicolumn{6}{|l|}{ Type of complication } \\
\hline Pressure ulcer ${ }^{\mathrm{a}}$ & 248 & 18 & 96 & 20 & 0.200 \\
\hline Urinary tract infection & 435 & 30 & 109 & 23 & 0.001 \\
\hline Cardiac complication & 191 & 13 & 85 & 18 & 0.018 \\
\hline Pneumonia & 79 & 6 & 65 & 14 & $<0.001$ \\
\hline Wound infection, superficial & 69 & 5 & 16 & 3 & 0.174 \\
\hline Wound infection, deep & 24 & 2 & 9 & 2 & 0.768 \\
\hline Acute renal failure & 32 & 2 & 10 & 2 & 0.416 \\
\hline Gastrointestinal bleeding & 27 & 2 & 9 & 2 & 0.993 \\
\hline Myocardial infarction & 49 & 3 & 18 & 4 & 0.729 \\
\hline Cerebrovascular lesion & 27 & 2 & II & 2 & 0.577 \\
\hline Pulmonary embolism & 14 & I & 5 & I & 0.899 \\
\hline Deep venous thrombosis & 17 & I & 4 & I & 0.899 \\
\hline \multicolumn{6}{|l|}{ Mortality } \\
\hline Mortality, 30 days & 136 & 10 & 74 & 15 & $<0.001$ \\
\hline Mortality, 0-4 months & 207 & 14 & 116 & 24 & $<0.001$ \\
\hline
\end{tabular}

Notes: *All P-values chi-square tests, women vs men. ${ }^{\text {aPressure ulcers grade I-IV }}$ developed during hospital stay. moderate cognitive dysfunction also sustain a high risk of developing complications ${ }^{19}$ that are even harder to identify in the clinical setting. ${ }^{18}$ In this study, we found that cognitive dysfunction increased the risk for all complications, such as UTI, pressure ulcer, cardiac complication, and pneumonia. We also found that mental impairment was the strongest predictor for pneumonia in men, besides having already present pulmonary disease. We therefore recommend the use of an instrument like the SPMSQ at the time of admission to identify these patients. We found that present pulmonary disease was associated with pneumonia and was also more common among men. It is known from previous studies that chest infection and cardiovascular disease are the most common postoperative complications after hip fracture and may lead to increased mortality. ${ }^{20-22}$ This is in accordance with our findings, which also show an increased mortality after a diagnosed pneumonia. Chatterton et $\mathrm{al}^{20}$ have also shown that male sex together with increasing age and comorbidity were associated with mortality during hospital stay as well as pneumonia and cardiovascular disease. The result from our study highlights further the need to also consider the sex

Table 3 Crude effect of preoperative variables on pressure ulcer, cardiac complication, and pneumonia

\begin{tabular}{|c|c|c|c|c|c|c|c|c|c|}
\hline \multirow[t]{2}{*}{ Binary variable } & \multicolumn{3}{|c|}{ Pressure ulcer } & \multicolumn{3}{|c|}{ Cardiac complication } & \multicolumn{3}{|c|}{ Pneumonia } \\
\hline & OR & $(95 \% \mathrm{Cl})$ & $P$ & OR & $(95 \% \mathrm{Cl})$ & $P$ & OR & $(95 \% \mathrm{Cl})$ & $P$ \\
\hline \multicolumn{10}{|l|}{ Baseline } \\
\hline Sex, male vs female ${ }^{a}$ & 1.2 & $(0.9-1.6)$ & 0.2 & 1.4 & $(1.1-1.9)$ & 0.016 & 2.7 & $(1.9-3.8)$ & $<0.001 *$ \\
\hline Age, $85-103$ vs $65-84^{a}$ & 1.4 & $(I . I-1.8)$ & $0.003^{*}$ & 1.3 & $(1.0-1.6)$ & 0.06 & 1.2 & $(0.8-1.7)$ & 0.3 \\
\hline Residence, institutional vs own home & $\mathrm{I} .4$ & $(I . I-I .8)$ & 0.012 & 0.8 & $(0.6-1.6)$ & 0.3 & 1.2 & $(0.8-1.8)$ & 0.3 \\
\hline $\begin{array}{l}\text { ADL, assisted vs independent }{ }^{\mathrm{a}} \\
\text { (Katz A-B vs } \mathrm{C}-\mathrm{G})\end{array}$ & 1.6 & $(1.2-2.0)$ & $0.00 I^{*}$ & 0.9 & $(0.7-1.2)$ & 0.5 & 1.4 & $(0.9-2.0)$ & 0.09 \\
\hline $\begin{array}{l}\text { Fracture type, extracapsular vs } \\
\text { intracapsular }^{a}\end{array}$ & 1.2 & $(1.0-1.5)$ & 0.1 & 0.9 & $(0.7-1.1)$ & 0.3 & $\mathrm{I} . \mathrm{I}$ & $(0.8-1.5)$ & 0.7 \\
\hline ASA classification, $3-4$ vs $1-2^{a}$ & 1.4 & $(I . I-I .8)$ & 0.013 & 2.2 & $(1.6-3.0)$ & $<0.001$ & 2.5 & $(1.6-3.7)$ & $<0.001$ \\
\hline $\begin{array}{l}\text { Time to surgery, } 0-1 \text { day } \\
\text { vs } 2 \text { or more days }\end{array}$ & 1.6 & $(1.3-2.1)$ & $<0.00 I^{*}$ & 1.7 & $(1.3-2.3)$ & $<0.00 I^{*}$ & 1.5 & $(1.0-2.1)$ & 0.045 \\
\hline \multicolumn{10}{|c|}{$\begin{array}{l}\text { vs } 2 \text { or more days } \\
\text { Presence vs absence of specific concomitant disease }\end{array}$} \\
\hline Cardiovascular disease & I.I & $(0.9-1.4)$ & 0.42 & 2.6 & $(1.9-3.5)$ & $<0.00 I^{*}$ & I.I & $(0.8-1.6)$ & 0.7 \\
\hline Stroke & 1.0 & $(0.8-1.4)$ & 0.9 & I.I & $(0.8-1.5)$ & 0.7 & $\mathrm{I} . \mathrm{I}$ & $(0.7-1.7)$ & 0.7 \\
\hline Pulmonary disease & 1.5 & $(1.1-2.0)$ & $0.006 *$ & 2.1 & $(1.5-2.8)$ & $<0.001$ & 2.7 & $(1.9-3.9)$ & $<0.00 I^{*}$ \\
\hline Renal disease & 1.2 & $(0.7-2.0)$ & 0.6 & 1.5 & $(0.9-2.6)$ & 0.6 & 1.2 & $(0.5-2.7)$ & 0.6 \\
\hline Diabetes & 0.8 & $(0.6-1.2)$ & 0.4 & 1.2 & $(0.8-1.8)$ & 0.3 & $\mathrm{I} . \mathrm{I}$ & $(0.6-1.7)$ & 0.9 \\
\hline Rheumatoid arthritis & 0.8 & $(0.5-1.3)$ & 0.3 & 1.2 & $(0.7-2.0)$ & 0.4 & I.I & $(0.6-2.1)$ & 0.8 \\
\hline Parkinson's disease & $\mathrm{I} .4$ & $(0.8-2.6)$ & 0.3 & 0.8 & $(0.4-1.7)$ & 0.5 & 1.9 & $(0.9-4.2)$ & 0.08 \\
\hline Malignancy & 1.3 & $(1.0-1.8)$ & 0.065 & 1.3 & $(1.0-1.9)$ & 0.08 & 1.3 & $(0.8-2.0)$ & 0.3 \\
\hline Cognitive function, $* *$ & 1.5 & $(1.2-2.0)$ & 0.001 & 1.6 & $(1.2-2.1)$ & $0.002^{*}$ & 2.4 & $(1.6-3-6)$ & $<0.00 I^{*}$ \\
\hline \multicolumn{10}{|l|}{ dysfunction vs normal ${ }^{a}$} \\
\hline \multicolumn{10}{|c|}{ Presence of additional complications and mortality } \\
\hline $\begin{array}{l}\text { Number of complications, } 2 \text { or } \\
\text { more vs } I^{a}\end{array}$ & 1.6 & $(1.2-2.0)$ & $<0.001$ & 2.6 & $(1.8-3.1)$ & $<0.001$ & 3.1 & $(2.0-4.7)$ & $<0.001$ \\
\hline Mortality within 30 days, yes vs no ${ }^{a}$ & 2.0 & $(1.5-2.8)$ & $<0.001$ & 3.9 & $(2.9-5.4)$ & $<0.001$ & 2.6 & $(1.7-3.9)$ & $<0.001$ \\
\hline Mortality $0-4$ months, yes vs no ${ }^{a}$ & 1.6 & $(1.2-2.2)$ & 0.001 & 2.5 & $(1.8-3.3)$ & $<0.001$ & 2.2 & $(1.5-3.2)$ & $<0.001$ \\
\hline
\end{tabular}

Notes: All P-values chi-square tests. *Variables still significant after adjusting for associated baseline variables. **According to the Short Portable Mental Status Questionnaire (0-7 vs 8-10). ${ }^{a}$ Reference group.

Abbreviations: ASA, American Society of Anesthesiologists; ADL, activities of daily living; $\mathrm{Cl}$, confidence interval; OR, odds ratio. 
Table 4 Logistic regression models of baseline variables associated with the most frequent complications

\begin{tabular}{|c|c|c|c|c|c|c|c|c|c|c|c|c|}
\hline & \multicolumn{3}{|c|}{ Urinary tract infection } & \multicolumn{3}{|c|}{ Pressure ulcer } & \multicolumn{3}{|c|}{ Cardiac complication } & \multicolumn{3}{|c|}{ Pneumonia } \\
\hline & OR & $(95 \% \mathrm{Cl})$ & $P$ & OR & $(95 \% \mathrm{Cl})$ & $P$ & OR & $(95 \% \mathrm{Cl})$ & $P$ & OR & $(95 \% \mathrm{Cl})$ & $P$ \\
\hline \multicolumn{13}{|l|}{ Sex } \\
\hline Female $^{\mathrm{b}}$ & 1 & & & & & & & & & 1 & & \\
\hline Male & 0.7 & $(0.5-0.9)$ & 0.003 & & & & & & & 2.4 & $(1.7-3.6)$ & $<0.001$ \\
\hline \multicolumn{13}{|l|}{ Age } \\
\hline $65-84^{b}$ & 1 & & & I & & & & & & & & \\
\hline$>85$ & 1.2 & $(0.0-1.5)$ & 0.034 & 1.4 & $(I . I-1.8)$ & 0.008 & & & & & & \\
\hline \multicolumn{13}{|l|}{ ADL } \\
\hline Independent ${ }^{\mathrm{b}}$ (Katz $\left.\mathrm{A}-\mathrm{B}\right)$ & & & & I & & & & & & & & \\
\hline Dependent (Katz C-G) & & & & 1.6 & $(1.2-2.1)$ & 0.001 & & & & & & \\
\hline \multicolumn{13}{|l|}{ Time-to-surgery } \\
\hline $0-I$ day $^{\mathrm{b}}$ & & & & I & & & I & & & & & \\
\hline I days & & & & 1.6 & $(1.2-2.1)$ & $<0.00 \mathrm{I}$ & 1.6 & $(1.2-2.2)$ & 0.001 & & & \\
\hline \multicolumn{13}{|l|}{ Cardiovascular disease } \\
\hline $\mathrm{No}^{b}$ & & & & & & & I & & & & & \\
\hline Yes & & & & & & & 2.2 & $(1.5-3.0)$ & $<0.001$ & & & \\
\hline \multicolumn{13}{|l|}{ Pulmonary disease } \\
\hline $\mathrm{No}^{b}$ & & & & 1 & & & 1 & & & 1 & & \\
\hline Yes & & & & 1.6 & $(1.2-2.2)$ & 0.003 & 2.0 & $(1.4-2.8)$ & $<0.001$ & 2.8 & $(1.8-4.2)$ & $<0.001$ \\
\hline \multicolumn{13}{|l|}{ Cognitive function } \\
\hline Normal (SPMSQ $\left.{ }^{\mathrm{a}} 8-10\right)^{\mathrm{b}}$ & & & & & & & 1 & & & I & & \\
\hline Dysfunction (SPMSQ 0-7) & & & & & & & 1.5 & $(I . I-2.0)$ & 0.006 & 2.5 & $(1.6-3.8)$ & $<0.001$ \\
\hline
\end{tabular}

Notes: ${ }^{a}$ The SPMSQ. ${ }^{\text {Reference variable. }}$

Abbreviations: $\mathrm{ADL}$, activities of daily living; $\mathrm{Cl}$, confidence interval; OR, odds ratio; SPMSQ, Short Portable Mental Status Questionnaire.

aspect when assessing the patients' risk for serious complications such as pneumonia or cardiac complications. Despite this knowledge, it seems that the care of these patients needs to be improved, for example, by taking into account male sex as a strong risk factor, considering a more active pre- and postoperative physiotherapeutic treatment, and starting antibiotic treatment sooner. Patients with cardiac disease need to be identified early to be able to consider a more active pre- and postoperative treatment. Under optimum conditions, there is a clear treatment algorithm that includes cardiac evaluation such as adjustment of fluid balance, hemoglobin, and saturation. Assessment by a cardiologist already at the emergency ward should be considered, also including a plan for follow-up during the hospital stay. In certain orthopedic clinics in Sweden, there is now a staff geriatrician, and this has been a valuable contribution to optimize the medical treatment of hip fracture patients.

Somewhat surprising, a previous history of stroke did not correlate with any complication, but identical results have also been reported previously. ${ }^{23}$ In the current study, UTI was identified if antibiotics were provided for this condition. UTI was found to be the most common complication, which is in line with several other studies. ${ }^{3,5,24}$ Still, the present incidence was even higher than reported by others. ${ }^{25,26}$ This may be explained by the fact that we followed all patients up to
4 months after the fracture. Time to surgery was associated with an increased incidence of pressure ulcers, which is consistent with prior findings. ${ }^{27-29}$ Furthermore, cardiac complications were associated with delayed time to surgery. Previous studies have shown the importance of reduced waiting time to surgery to increase the patients possibility to return to independent living, ${ }^{29}$ to reduce hospital stay, and possibly decrease complication rate and mortality. ${ }^{30}$ The Swedish Board has placed a major focus on this issue and demands an annual account of the number of patients with hip fracture who have had surgery within 24 hours. This time limit is also linked to economic compensation, and this could be one way to raise the awareness of the importance of providing a fast track to surgery for patients with hip fracture. ${ }^{12}$ More than half the number of patients in our study had a cardiovascular disease. This comorbidity could require optimization of the patients' health condition before surgery, which in turn could lead to a delay. The question is how to balance these two demands. In a recent review article, it was suggested that we need a more active approach in the preoperative risk assessment, medical and orthopedic comanagement, and perioperative monitoring to be able to operate on the patient within time limits and as safe as possible. ${ }^{31}$ We agree that the preoperative risk assessment is of importance and that it should be carried out systematically using a validated and 
manageable instrument. In a study evaluating the impact of surgical timing on surgical outcomes, the orthopedic Physiological and Operative Severity Score for the enUmeration of Mortality and Morbidity (POSSUM) was used. The study showed a small improvement of the physiological score from admittance to before surgery and that the POSSUM may be used to identify patients with a higher risk for morbidity if surgery was delayed over 24 hours. ${ }^{32}$

The strengths of this study are the large number of patients as well as the inclusion of patients with cognitive dysfunction. Cognitive dysfunction is often an exclusion criterion in prospective studies of hip fracture patients. The other strengths of the study are the use of validated instruments and protocols, for example, as in our use of the SAFHE, as well as the thorough review of all the charts. These were performed by specially trained research nurses who also interviewed all the patients. There are several limitations of the study. The study did not include current medications, laboratory parameters, or secondary causes of osteoporosis that could have further contributed to the assessment of the patients' risk of mortality. A possible limitation was the missing data which, however, only comprised $1.4 \%$ of the patients and therefore is unlikely to affect the outcome.

\section{Conclusion}

Before surgery, men with hip fracture already have a poorer health status and higher comorbidity rate than women, resulting in a twofold increased risk of pneumonia and also an increased risk of cardiac complications. Cognitive dysfunction and present pulmonary disease were related to pneumonia in men. Delayed surgery seems to increase the risk for cardiac complications. It is important to consider the sex perspective early on together with cardiopulmonary comorbidity and cognitive dysfunction to be able to counteract serious complications that may lead to death.

\section{Acknowledgment}

The authors thank the Stockholm Hip Fracture Group, including Anita Söderqvist, Åsa Norling, Paula Kelly-Pettersson, Kristina Källman, Gustaf Neander, Nils Dalén, Eva Samnegård, Maria Sääf, Jan Tidermark, and Amer Al-Ani for their help in conducting the study.

This study was supported by the Stockholm County Research for Clinical Studies (EXPO 1999) and by grants from Sophiahemmet University College and Sophiahemmet Foundation for Clinical Studies. The funding sources played no role in the design, method, subject recruitment, data collection, analysis, or preparation of this paper.

\section{Disclosure}

The authors report no conflicts of interest in this work.

\section{References}

1. Thorngren KG, Hommel A, Norrman PO, Thorngren J, Wingstrand H. Epidemiology of femoral neck fractures. Injury. 2002;(33 Suppl 3): C1-C7.

2. Löfgren S, Ljunggren G, Brommels M. No ticking time bomb: hospital utilisation of 28,528 hip fracture patients in Stockholm during 1998-2007. Scand J Public Health. 2010;38(4):418-425.

3. Hedström M, Gröndal L, Ahl T. Urinary tract infection in patients with hip fractures. Injury. 1999;30(5):341-343.

4. Giusti A, Barone A, Razzano M, Pizzonia M, Olivieri M, Pioli G. Predictors of hospital readmission in a cohort of 236 elderly discharged after surgical repair of hip fracture: one-year follow-up. Aging Clin Exp Res. 2008;20(3):253-259.

5. Merchant RA, Lui KL, Ismail NH, Wong HP, Sitoh YY. The relationship between postoperative complications and outcomes after hip fracture surgery. Ann Acad Med Singapore. 2005;34(2):163-168.

6. Vestergaard P, Rejnmark L, Mosekilde L. Increased mortality in patients with a hip fracture-effect of pre-morbid conditions and post-fracture complications. Osteoporos Int. 2007;18(12):1583-1593.

7. Wehren LE, Hawkes WG, Orwig DL, Hebel JR, Zimmerman SI, Magaziner J. Gender differences in mortality after hip fracture: the role of infection. J Bone Miner Res. 2003;18(12):2231-2237.

8. Panula J, Pihlajamäki H, Mattila V, et al. Mortality and cause of death in hip fracture patients aged 65 or older. A population based study. BMC Musculoskelet Disord. 2011;12:105.

9. Endo Y, Aharonoff GB, Zuckerman JD, Egol KA, Koval KJ. Gender differences in patients with hip fracture: a greater risk of morbidity and mortality in men. J Orthop Trauma. 2005;19(1):29-35.

10. Samuelsson B, Hedström M, Ponzer S, et al. Gender differences and cognitive aspects on functional outcome after a hip fracture-a 2 years follow up study of 2,134 patients. Age Ageing. 2009;38(6):686-692.

11. Söderqvist A, Ekström W, Ponzer S, et al. Prediction of mortality in elderly patients with hip fractures: a two-year prospective study of 1,944 patients. Gerontology. 2009;55(5):496-504.

12. Swedish National Board of Health and Welfare. Socialstyrelsens riktlinjer för vård och behandling av höftfraktur. Stockholm, Sweden: Swedish National Board of Health and Welfare; 2003. Swedish.

13. Parker MJ, Currie CT, Thorngren KG. Standardised audit of hip fractures in Europe. Hip Int. 1998;8:10-15.

14. Katz S, Ford AB, Moskowitz RW, Jackson BA, Jaffe MW. Studies of illness in the aged. The index of ADL: a standardized measure of biological and psychosocial function. JAMA. 1963;185:914-919.

15. Owens WD, Felts JA, Spitznagel EL Jr. ASA physical status classifications: a study of consistency of ratings. Anesthesiology. 1978;49(4): 239-243.

16. Pfeiffer E. A short portable mental status questionnaire for the assessment of organic brain deficit in elderly patients. J Am Geriatr Soc. 1975; 23(10):433-441.

17. European Pressure Ulcer Advisory Panel. Pressure Ulcer Prevention/ Treatment Guidelines. Oxford, UK: EUPAP; 1999. Available from: http://www.npuap.org/wp-content/uploads/2014/08/Updated-1016-14-Quick-Reference-Guide-DIGITAL-NPUAP-EPUAP-PPPIA16Oct2014.pdf. Accessed June 5, 2015.

18. Söderqvist A, Strömberg L, Ponzer S, Tidermark J. Documenting the cognitive status of hip fracture patients using the Short Portable Mental Status Questionnaire. J Clin Nurs. 2006;15(3):308-314.

19. Stromberg L, Lindgren U, Nordin C, Ohlén G, Svensson O. The appearance and disappearance of cognitive impairment in elderly patients during treatment for hip fracture. Scand J Caring Sci. 1997;11(3): 167-175.

20. Chatterton BD, Moores TS, Ahmad S, Cattell A, Roberts PJ. Cause of death and factors associated with early in-hospital mortality after hip fracture. Bone Joint J. 2015;97-B(2):246-251. 
21. Boereboom FT, Raymakers JA, Duursma SA. Mortality and causes of death after hip fractures in The Netherlands. Neth J Med. 1992;41(1-2):4-10.

22. Roche JJ, Wenn RT, Sahota O, Moran CG. Effect of comorbidities and postoperative complications on mortality after hip fracture in elderly people: prospective observational cohort study. BMJ. 2005; 331(7529):1374.

23. Youm T, Aharonoff G, Zuckerman JD, Koval KJ. Effect of previous cerebrovascular accident on outcome after hip fracture. J Orthop Trauma. 2000;14(5):329-334.

24. Pedersen SJ, Borgbjerg FM, Schousboe B, et al. A comprehensive hip fracture program reduces complication rates and mortality. J Am Geriatr Soc. 2008;56(10):1831-1838.

25. Kamel HK. The frequency and factors linked to a urinary tract infection coding in patients undergoing hip fracture surgery. J Am Med Dir Assoc. 2005;6(5):316.

26. Norris R, Parker M. Diabetes mellitus and hip fracture: a study of 5,966 cases. Injury. 2011;42(11):1313-1316.

27. Lindholm C, Sterner E, Romanelli M, et al. Hip fracture and pressure ulcers - the Pan-European Pressure Ulcer Study - intrinsic and extrinsic risk factors. Int Wound. 2008;5(2):315-328.
28. Hommel A, Ulander K, Björkelund KB, Norrman PO, Wingstrand H, Thorngren KG. Influence of optimised treatment of people with hip fracture on time to operation, length of hospital stay, reoperations and mortality within 1 year. Injury. 2008;39(10):1164-1174.

29. Al-Ani AN, Samuelsson B, Tidermark J, et al. Early operation on patients with a hip fracture improved the ability to return to independent living. A prospective study of patients. J Bone Joint Surg Am. 2008; 90(7):1436-1442.

30. Khan SK, Kalra S, Khanna A, Thiruvengada MM, Parker MJ. Timing of surgery for hip fractures: a systematic review of 52 published studies involving 291,413 patients. Injury. 2009;40(7):692-697.

31. Nicholas JA. Preoperative optimization and risk assessment. Clin Geriatr Med. 2014;30(2):207-218.

32. Hapuarachchi KS, Ahluwalia RS, Bowditch MG. Neck of femur fractures in the over 90s: a select group of patients who require prompt surgical intervention for optimal results. J Orthop Traumatol. 2014; 15(1):13-19.
Clinical Interventions in Aging

\section{Publish your work in this journal}

Clinical Interventions in Aging is an international, peer-reviewed journal focusing on evidence-based reports on the value or lack thereof of treatments intended to prevent or delay the onset of maladaptive correlates of aging in human beings. This journal is indexed on PubMed Central, MedLine,

\section{Dovepress}

CAS, Scopus and the Elsevier Bibliographic databases. The manuscript management system is completely online and includes a very quick and fair peer-review system, which is all easy to use. Visit http://www.dovepress. com/testimonials.php to read real quotes from published authors. 